Revista de Matemática: Teoría y Aplicaciones 1996 3(1) : 1-10

CIMPA - UCR ISSN: 1409-2433

\title{
LA FORMA HEXAGONAL REGULAR DE LAS CÉLULAS DE LAS ABEJAS COMO SOLUCIÓN DE ALGUNOS PROBLEMAS DE ÓPTIMO*
}

\author{
IOAN MUNTEAN ${ }^{\dagger}$
}

15 Julio 2021

\begin{abstract}
Resumen
La economía de cera y la resistencia del panal, así como otras hipótesis plausibles (eliminación de espacios vacíos entre células cilíndricas y emulación aproximada del cuerpo de la abeja) conducen al primer problema de óptimo: entre todos los polígonos con $n \geq 3$ lados circunscritos a un círculo de radio dado, determinar el polígono $P_{n}^{*}$ que tiene perímetro más pequeño. Este es un problema de extremos con una condición isogonal que se resuelve por el método de multiplicadores de Lagrange. Se demuestra que $P_{n}^{*}$ es polígono regular y que $n \in\{3,4,6\}$. Finalmente, otro problema de mínimo conduce al resultado $n=6$.
\end{abstract}

Palabras clave: optimización; panales de abejas; condición isogonal.

\begin{abstract}
Wax compression and honeycomb resistance, and some other hypothesis as well (elimination of empty spaces between cylindrical cells and approximate emulation of bees bodies) drive to the first optimization problem: among all polygons with $n \geq 3$ sides circumscribed into a circle with a given radius, determine the polygon $P_{n}^{*}$ with the smallest perimeter. This extrema problem with an isogonal condition is solved with a Lagrange multipliers method. It is proven that $P_{n}^{*}$ is a regular polygon and $n \in\{3,4,6\}$. Finally, another minimum problem drives to $n=6$.
\end{abstract}

Keywords: optimization; honeycombs; isogonal condition.

Mathematics Subject Classification: 49M30.

\footnotetext{
* Este constituye el último artículo del Profesor Muntean, ver obituario al final del texto.

${ }^{\dagger}$ Facultatea de Matematica si Informatica, Universitattea "Babes-Bólyai" , Str. M. Kogalniceanu 1, 3400 Cluj-Napoca, Rumania.
} 


\section{Introducción}

Los panales de abejas (Apis mellifera $L$ ) son pares de capas adyacentes construidos de cera y formados de células o celdas pequeñas donde ellas colocan la miel y el polen (bajo la forma de pasta así como los huevos que luego desarrollan las larvas. Las celdas son dos a dos congruentes y tienen forma poliedral: es decir son prismas hexagonales regulares que se cierran con un techo piramidal edificado de tres superficies romboidales congruentes. Las entradas en las celdas situadas en la misma parte del panal generan una red de hexágonos regulares, más exactamente un enrejado regular y completo de superficies hexagonales por ambas caras del panal (ver Figura 1).

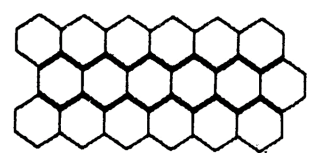

Figura 1: Red de hexágonos regulares del panal.

Una investigación más atenta constata que debido a la forma piramidal del techo de la célula, así como la necesidad de eliminar los huecos y también para que la célula ofrezca mayor resistencia, ninguna célula de una capa está opuesta exactamente a otra de la otra capa, como si una fuera prolongación de la otra; el eje de cada célula de una capa estará situado en la prolongación de la arista común a tres celdas vecinas de la capa opuesta.

Las investigaciones efectuadas por el alemán H. Vogt [13] demuestran que las dimensiones medias de una célula de abeja son las siguientes: el lado del hexágono es de 2,71 $\mathrm{mm}$, la longitud de las aristas grandes del prisma es de 11,3 $\mathrm{mm}$, y el grueso de las paredes de las células es de 0,08 $\mathrm{mm}$; en las consideraciones matemáticas de más abajo el grueso de las paredes se va a despreciar.

La estructura de los panales, descrita más arriba y llamada por algunos autores geometría o arquitectura de las abejas, no puede ser enteramente comprendida sin un estudio post - factum de los motivos en los cuales se apoya el proyecto de tal estructura. El presente trabajo intenta poner en claro estos motivos y, con base en ellos, dar una solución para el primero de los siguientes problemas relativos a los panales de abejas:

A. ¿Por qué las abejas dieron a las células la forma hexagonal regular?

B. ¿Por qué las abejas adoptaron como valores de los ángulos de las superficies romboidales mencionadas precisamente los de las medidas de G.F. Máraldi [4]: 109 $28^{\prime}$ y $70^{\circ} 32^{\prime}$ ?

Una solución al problema B va a ser dada en [5]. La sección 2 del presente trabajo contiene un prefacio histórico del problema A, en la sección 3 se enunciará un problema isogonal auxiliar de óptimo denotado con $\mathrm{C}$, en la sección 4 se resuelve el problema $\mathrm{C}$ y en la última sección, como una síntesis, se llega a la forma hexagonal regular de las células de abejas como solución al problema A.

\section{Historia del problema A}

La diligencia de las abejas, así como su "castidad" y "eternidad", fueron expresadas por el poeta latino Publius Vergilius Maro, conocido como Virgilio (70-19 d.C.) en el libro IV del poema, suyo Las Geórgicas, escrito entre los años 39-29 d.C.: 
"Tantus amor floram, et generandi gloria mellis" (Tanto amor trae una flor, cuanta miel preciosa tiene).

En el mismo período, el erudito Marcus Terentius Varro (117-27 d.C.) observa en Rerum rusticarum, escrito alrededor del año $36 \mathrm{~d}$.C, que las células de las abejas tienen todas forma hexagonal y que su "hábil colocación en panales" tiene como efecto no solamente una economía de cera, sino también la eliminación completa de espacios vacíos. También, Varro afirma que, "así como se ve en geometría el hexágono regular inscrito en un circulo comprende una área mayor que otra figura con menos lados", así aparece el primer intento de resolver el problema A.

Después de aproximadamente 300 años la misma solución del problema A fue precisada por el geómetra griego Pappus de Alejandría (siglo III a.C.), que en el prólogo "Sobre la inteligencia de las abejas" en el libro V de su obra base "Collectiones Mathematícae" [8], Vol.I, pag. 237, escribe:

[...] aunque los dioses han provisto solamente al hombre de razón, ellos le dieron a los animales el instinto. Asi las abejas no fabrican la miel en forma negligente dondequiera, al azar, sino recogiendo el néctar de las flores más hermosas. Ellas construyen primero vasos, todos iguales, semejantes, pegados entre ellos, en los cuales depositan la miel. Pero sólo tres figuras regulares: triángulo, cuadrado y hexágono satisfacen estas condiciones y las abejas han elegido para construir los panales la figura que tiene más vértices, pues concluyeron que va a contener más miel que las otras para un consumo igual de cera (ver también [1] pag.40-41).

El célebre astrónomo Johannes Kepler (1571-1630), descubridor de las leyes de los movimientos de los planetas alrededor del sol y buen conocedor de la obra de Pappus, pero no lo suficientemente convencido de los argumentos de Varro, explica la preferencia de las abejas por la sección hexagonal regular por el hecho de que la densidad de la red de las paredes de cera - y entonces la densidad del panal construido de células hexagonales regulares adyacentes - es mayor que la densidad que tendría un panal formado de células cuadradas o triangulares equiláteras [3] (ver también [11]).

El punto de vista de Pappus fue sostenido por autores que publicaron en nuestro siglo como E.G. Togliatti [12], pag.1-71, e Ion Ionescu [2], pag. 137.

Otros aspectos históricos del problema A se hallan en los artículos [1] y [15].

\section{El problema isogonal $\mathrm{C}$}

Las investigaciones palenteológicas demuestran que los insectos recolectores de néctar y polen aparecieron hace aproximadamente 150-100 millones de años, y las abejas en sociedad que almacenan reservas de miel aparecieron hace aproximadamente 20-10 millones de años ([14] pag. 19). Un grupo de investigadores de la Universidad Politécnica de California, Estados Unidos, descubrieron, en la primera mitad de 1995, una bacteria conservada perfectamente en el cuerpo de una abeja muerta hace aproximadamente 40-25 millones de años y hallada en la resina de un árbol petrificado, la bacteria aún viva produjo un antibíotico natural (ver [16] pag. 7). 

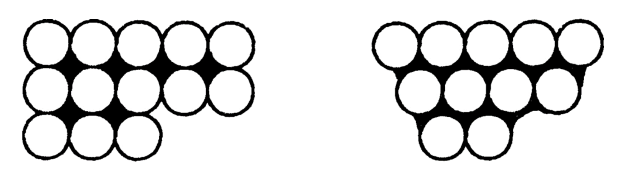

Figura 2: Forma para consolidar la resistencia del panal.

Es plausible que en decenas de miles de años de existencia, la "concepción" de las abejas sobre la construcción de las células evolucionó a formas cada vez más perfeccionadas, en el sentido que precisaremos más abajo. Teniendo en cuenta la forma aproximadamente cilíndrica tanto del cuerpo de las abejas (que tienen que tener acceso a cada célula), como del cuerpo de las larvas (que pasan su vida en las células hasta el estadio de adultos), las abejas pudieron ser "tentadas" a producir células circulares. Para consolidar la resistencia del panal, las células circulares vecinas necesitan ser tangentes y dispuestas como se ve, por ejemplo, en la Figura 2; es claro que la última configuración da al panal una resistencia mayor que la primera -este hecho fue precisado por Kepler [3] pag. 721. Las abejas renunciaron a la anterior configuración pues ella genera muchos espacios vacíos entre las paredes circulares de las células. (Precisamos que en las dos capas de un panal se hallan cerca de 10000 células, de aquí que el espacio no utilizado sería, demasiado grande.) Se sabe que la Apis Florea, una especie de abeja pequeña que vive en Asia del sur-este, construye aun hoy día tanto células hexagonales, como circulares (ver [14] pag.55).

Otro argumento en favor de renunciar a la forma circular consta en que, para economizar cera las abejas construyen los panales de tal manera que cada porción de la superficie lateral de una célula sea la pared común para dos células vecinas, y la célula misma sea un recipiente o cavidad convexa (de otra manera en la célula quedarían espacios grandes no ocupados por el cuerpo de la abeja y difícilmente accesibles en el proceso de construcción, o para depositar miel, evacuar o limpiar la célula).

Esto último es realizable solamente si la forma de las células, es decir la sección plana perpendicular al eje de la célula interseca la superficie de la célula en un polígono convexo. Para las abejas constructoras es cómodo que pasando de la edificación de una célula a otra, los polígonos correspondientes tengan un mismo número de lados; luego, también que permita el acceso de la abeja a toda, la célula, para esto debe emular bien el cuerpo de esta y utilizar una cantidad mínima de cera en el proceso de construcción. Es claro que los argumentos nuestros contrastan con los citados por Varro y Pappus, así como los puntos de vista de otros autores mencionados en la sección 2 .

Pasamos ahora a formular matemáticamente el problema que se desprende de las consideraciones precedentes. Denotaremos con $\prod_{n}$ el conjunto de todos los polígonos con un mismo número dado $n \geq 3$ de lados, que están circunscritos en un círculo de radio $r>O$ y con $\lambda\left(P_{n}\right)$ el perímetro de un polígono $P_{n} \in \prod_{n}$. El problema se denota con $\mathrm{C}$ y se enuncia así:

(C) Determinar un polígono $P_{n}^{*} \in \Pi_{n}$, con la propiedad:

$$
\lambda\left(P_{n}^{*}\right) \leq \lambda\left(P_{n}\right)
$$

cualquiera que sea $P_{n} \in \Pi_{n}$. 


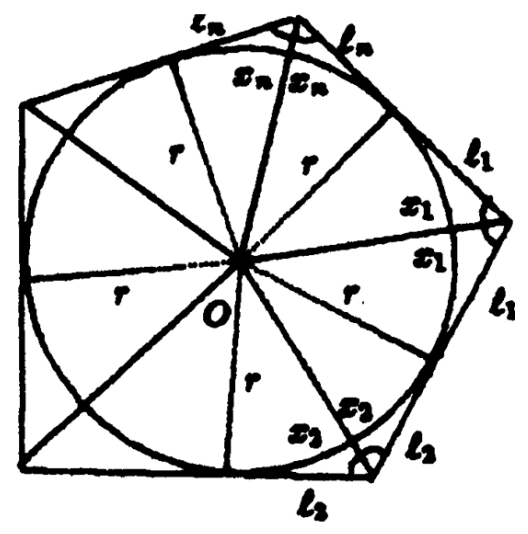

Figura 3: Forma para consolidar la resistencia del panal.

Cada polígono $P_{n} \in \Pi$ está unívocamente determinado (exceptuando una rotación alrededor del centro $O$ del círculo) de las medidas $x_{1}, \ldots, x_{n}$ de los semiángulos suyos, podemos entonces identificar a $P_{n}$ con $x_{1}, \ldots, x_{n}$. De la Figura 3 tenemos

$$
\tan x_{1}=\frac{r}{l_{1}}, \ldots, \tan x_{n}=\frac{r}{l_{n}}
$$

entonces

$$
l_{1}=\frac{r}{\tan x_{1}}, \ldots, l_{n}=\frac{r}{\tan x_{n}}
$$

también, tiene lugar la condición "isogonal"

$$
x_{1}+\ldots+x_{n}=\pi\left(\frac{n}{2}-1\right)
$$

aquí $l_{i}>0$ y $0<x_{i}<\frac{\pi}{2}, i \in\{1, \ldots, n\}$.

Para $P_{n}=\left(x_{1}, \ldots, x_{n}\right) \in \Pi_{n}$ tenemos

$$
\lambda\left(P_{n}\right)=\sum_{i=1}^{n} 2 l_{i}=\sum_{i=1}^{n} \frac{2 r}{\tan x_{i}}
$$

\section{Una solución al problema $\mathrm{C}$}

El problema $\mathrm{C}$ es un problema de mínimo para la función $f$ : Dlongrightarrow $R$, dada por (2):

$$
f\left(x_{1}, \ldots, x_{n}\right)=\lambda\left(P_{n}\right)=\sum_{i=1}^{n} \frac{2 r}{\tan x_{i}},
$$

donde $(D=] 0, \frac{\pi}{2}[x \ldots x] 0, \frac{\pi}{2}\left[\right.$, con la restricción $g\left(x_{1}, \ldots, x_{n}\right)=0$, donde

$$
g\left(x_{1}, \ldots, x_{n}\right)=x_{1}+, \ldots,+x_{n}-\pi\left(\frac{n}{2}-1\right) .
$$


En lo que sigue, vamos a dar a este problema una solución basada en el método de multiplicadores de Lagrange y en el estudio de la diferencial segunda del lagrangeano asociado (ver [6], pag. 281-289; [7], pag. 660-665 y [9], pag. 22-30).

Sea $D$ una parte abierta del espacio euclídeo $R_{n}, f, g: D \longrightarrow \mathbb{R}$ dos funciones con derivadas parciales del orden dos continuas en una vecindad de un punto $x^{*}=\left(x_{1}^{*}, \ldots, x_{n}^{*}\right) \in D$ y admitimos que

$$
\left(\frac{\partial g}{\partial x_{1}}\left(x^{*}\right), \ldots, \frac{\partial g}{\partial x_{n}}\left(x^{*}\right)\right) \neq 0 .
$$

Denotamos $h_{\lambda}=f+\lambda g, \lambda \in \mathbb{R}, \mathrm{y}$

$$
E=\left\{k=\left(k_{1}, \ldots, k_{n}\right) \in R^{n}: \sum_{i=1}^{n} \frac{\partial g}{\partial x_{i}}\left(x^{*}\right) k_{i}=0\right\} .
$$

El siguiente teorema contiene una condición necesaria para extremos con condiciones.

Teorema 4.1 Si $x^{*} \in D$ es un punto de mínimo para $f$ con la condición $g\left(x_{1}, \ldots, x_{n}\right)=0$, entonces existe un $\lambda \in \mathbb{R}$ tal que

$$
\frac{\partial h_{\lambda}}{\partial x_{1}}\left(x^{*}\right)=0, \ldots, \frac{\partial h_{\lambda}}{\partial x_{n}}\left(x^{*}\right)=0
$$

Recíprocamente, admitiendo que además, $f$ y $g$ poseen derivadas parciales de orden tercero continuas en una vecindad de $x^{*}$ entonces tienen lugar las siguientes condiciones suficientes de óptimo.

Teorema 4.2 Si $x^{*} \in D$ y $\lambda \in \mathbb{R}$ verifican (5) y si la función $J: E \times E \longrightarrow \mathbb{R}$, definida por:

$$
J\left(K, K^{\prime}\right)=\sum_{i, j=1}^{n} \frac{\partial^{2} h_{\lambda}}{\partial x_{i} \partial x_{j}}\left(x^{*}\right) k_{i} k_{j}^{\prime}
$$

$k=\left(k_{1}, \ldots, k_{n}\right) \in E, k^{\prime}=\left(k_{1}^{\prime}, \ldots, k_{n}^{\prime}\right) \in E$ positivamente definida (la diferencial de orden dos de $\left.h_{\lambda}\right)$, entonces $x^{*}$ es el punto de mínimo para $f$ con la condición $g\left(x_{1}, \ldots, X_{n}\right)=0$.

El problema C halla, una solución en el siguiente teorema.

Teorema 4.3 Un polígono $P_{n}^{*} \in \Pi_{n}$ es solución del problema $C$, si y solo si, $P_{n}^{*}$ es polígono regular.

Demostración. Admitimos que $P_{n}^{*}$ es solución del problema C, es decir, según (1) y (2) el vector $x^{*}=\left(x_{1}^{*}, \ldots, x_{n}^{*}\right) \in D$, del cual los componentes son la mitad de las medidas de triángulos de $P_{n}^{*}$, es punto de mínimo para la función $f$, dada por (3), con la condición $g\left(x_{1}, \ldots, x_{n}\right)=O$, donde $g$ es la función definida por $(4)$. Como $\frac{\partial g}{\partial x_{1}}(x)=1$, para todo $i \in\{1, \ldots, n\}$ y para todo $x \in D$, por el teorema 4.1 se sigue que existe un $\lambda \in \mathbb{R}$ tal que

$$
0=\frac{\partial h_{\lambda}}{\partial x_{i}}\left(x^{*}\right)=\frac{-2 r}{\operatorname{sen}^{2} x_{i}^{*}}+\lambda, i \in\{1, \ldots, n\}
$$


de donde deducimos que $\lambda>O$ y que $\sin x_{i}^{*}=\left(\frac{2 r}{\lambda}\right)^{\frac{1}{2}}, i \in\{1, \ldots, n\}$. Denotando con $x_{0}^{*}$ el valor común de los números $x_{1}^{*}, \ldots, x_{n}^{*}$, es decir $x_{0}^{*}=\arcsin \left(\frac{2 r}{\lambda}\right)^{\frac{1}{2}}$, de (4), (1) y (6) obtenemos

$$
x_{1}^{*}=\ldots=x_{n}^{*}=x_{0}^{*}=\pi\left(\frac{1}{2}-\frac{1}{n}\right)
$$

y

$$
\lambda=\frac{2 r}{\sin ^{2} \pi\left(\frac{1}{2}-\frac{1}{n}\right)}=\frac{2 r}{\cos ^{2} \frac{\pi}{n}}
$$

De (7) resulta que $P_{n}^{*}=\left(x_{1}^{*}, \ldots, x_{n}^{*}\right)$ es polígono regular.

Recíprocamente, admitimos que $P_{n}^{*}=\left(x_{1}, \ldots, x_{n}^{*}\right) \in \Pi_{n}$ es un polígono regular, entones $2 x_{1}^{*}=\ldots=2 x_{n}^{*}$; denotamos con $2 x_{0}^{*}$, el valor común a los últimos números, entonces las desigualdades (6) y (7) tienen lugar, si se tiene en cuenta (1) y se define el número $\lambda$ por (8). Como para el punto $x^{*}=\left(x_{0}^{*}, \ldots, x_{0}^{*}\right) \in D$ tenemos

$$
\begin{gathered}
\frac{\partial^{2} h_{\lambda}}{\partial x_{i}^{2}}\left(x^{*}\right)=\frac{4 r}{\sin ^{3} x_{0}^{*}} \cos x_{0}^{*}=\frac{4 r \cos \left(\frac{\pi}{2}-\frac{\pi}{n}\right)}{\sin ^{3}\left(\frac{\pi}{2}-\frac{\pi}{n}\right)}=\frac{4 r \sin \frac{\pi}{n}}{\cos ^{3} \frac{\pi}{n}} \\
\frac{\partial^{2} h_{\lambda}}{\partial x_{i} \partial x_{j}}\left(x^{*}\right)=0
\end{gathered}
$$

cuando $i \neq j$, resulta que

$$
J\left(K, K^{\prime}\right)=\sum_{i=1}^{n} \frac{\partial^{2} h_{\lambda}}{\partial x_{i}^{2}}\left(x^{*}\right) k_{i}^{2}=\frac{4 r \sin \frac{\pi}{n}}{\cos ^{3} \frac{\pi}{n}}\left[\sum_{i=1}^{n-1} k_{i}^{2}+\left(\sum_{i=1}^{n-1} k_{i}\right)^{2}\right]>0 .
$$

Para todo $k=\left(k_{1}, \ldots, k_{n}\right) \in E$ con $k \neq 0$. En nuestro caso $E=\left\{\left(k_{1}, \ldots, k_{n}\right) \in R^{n}\right.$ : $\left.\sum_{i=1}^{n} \frac{\partial g}{\partial x_{i}}\left(x^{*}\right) k_{i}=0\right\}$

$=\left\{\left(k_{1}, \ldots, k_{n}\right) \in R^{n}: \sum_{i=1}^{n} k_{i}=0\right\}$.

Así la función $J$ es positiva definida en $E$, entonces por el teorema 4.2, el punto $x^{*}=$ $\left(x_{0}, \ldots, x_{0}^{*}\right)$, dado por $(7)$, es punto de mínimo para $f$ con la condición $g\left(x_{1}, \ldots, x_{n}\right)=0$. Por consiguiente, el polígono regular $P_{n}^{*}=\left(x_{0}^{*}, \ldots, x_{0}^{*}\right) \in D$ es solución del problema C.

\section{Forma hexagonal regular de la células de abejas}

Según lo establecido hasta ahora, los contornos de las células de un panal generan una red de polígonos regulares, dos a dos congruentes, con el mismo número $n \geq 3$ de lados, sin huecos entre las placas limitadas por el polígono y sin superposiciones en el interior de estas placas.

Denotaremos con $S\left(P_{n}\right)$ la superficie poligonal o placa cerrada y acotada, de la cual la frontera es el polígono $P_{n} \in \Pi_{n}$, entonces $S\left(P_{n}\right)$ es la envolvente convexa de $P_{n}$. Llamaremos red poligonal de tipo $n \geq 3$ cualquier colección $\mathcal{R}_{n}$, de polígonos regulares con $n \geq 3$ lados, dos a dos congruentes y sin superposiciones de los interiores de las superficies poligonales determinadas por este polígono. Denotaremos con $\mathcal{L}_{n}$ la reunión de todas las 
superficies poligonales determinadas por los polígonos que pertenecen a una red poligonal $\mathcal{R}_{n}$ de tipo $n \geq 3$. El siguiente teorema fue intuido también por otros autores, por ejemplo Ion Ionescii [2], pag. 135-136.

Teorema 5.1 Si $\mathcal{R}_{n}$ es una red poligonal de tipo $n \geq 3$ e interior $m \geq 3$ los polígonos de $\mathcal{R}_{n}$ tienen un vértice común perteneciendo al interior de la reunión $\mathcal{L}_{n}$ entonces $m \in$ $\{3,4,6\}$ y $n \in\{3,4,6\}$.

Demostración. Como existe un punto $p_{o}$ interior al conjunto $\mathcal{L}_{n}$ los $m$ polígonos de $\mathcal{R}_{n}$, que tienen un vértice común en $p_{o}$, van a tener la suma de las medidas de los ángulos que se intersecan en $p_{o}$, igual a $2 \pi$ radianes. Resulta que la medida de cada uno de estos ángulos es $\frac{2 \pi}{m}$ radianes, pues los polígonos mencionados son regulares, dos a dos congruentes y sin superposiciones en los interiores de las superficies poligonales determinadas por ellas.

Por otra parte, la suma de las medidas de los ángulos de cada uno de los $m$ polígonos es igual, con base en (1), a $\pi n-2 \pi=\pi(n-2)$ radianes, entonces cada ángulo de un tal polígono tiene medida igual a $\frac{\pi(n-2)}{n}$ radianes. De aquí y de lo precedente se obtiene:

$$
\frac{\pi(n-2)}{n}=\frac{2 \pi}{m} \text { ó } n=2+\frac{4}{m-2} \text {. }
$$

Como $n \geq 3$ es entero, la última igualdad de (9) prueba que también debe ser entero, entonces $m \in\{3,4,6\}$. La misma igualdad implica también $n \in\{3,4,6\}$.

De (2) y (7) deducimos que el perímetro $\lambda\left(P_{n}^{*}\right)$ de un polígono regular $P_{n}^{*} \in \Pi_{n}$ es:

$$
\lambda\left(P_{n}^{*}\right)=2 n r . \tan \frac{\pi}{n} \text {. }
$$

Se dice que la red poligonal $\mathcal{R}_{n_{o}}$ de un tipo $n_{o} \geq 3$ es minimal si $\lambda\left(P_{n_{o}}^{*}\right) \leq \lambda\left(P_{n}^{*}\right)$ para cualquier entero $m \geq 3$.

Teorema 5.2 Una red poligonal $\mathcal{R}_{n_{o}}$ de un tipo $n_{o} \geq 3$ del teorema 5.1 es minimal si $y$ sólo si $n_{o}=6$.

Demostración. De (10) tenemos

$$
\lambda\left(P_{6}^{*}\right)=4 r \sqrt{3}<8 r=\lambda\left(P_{4}^{*}\right)<6 r \sqrt{3}=\lambda\left(P_{3}^{*}\right)
$$

lo cual, con base en el teorema 5.1, termina la demostración.

\section{Observaciones}

a) El teorema 5.2 prueba que entre las células prismáticas rectas con contorno triangular equilátero, cuadrado o hexagonal regular, el que tiene área lateral más pequeña es la, célula con contorno hexagonal regular. Esta última, célula va a tener a su vez, el menor volumen, pues el área $\alpha\left(S\left(P_{n}^{*}\right)\right)$ tiene una superficie poligonal $S\left(P_{n}^{*}\right)$ delimitada por un polígono regular $P_{n}^{*}=\left(x_{1}^{*}, \ldots, x_{n}^{*}\right)$, está dada por

$$
\alpha\left(S\left(P_{n}^{*}\right)\right)=\sum_{i=1}^{n} \frac{r^{2}}{\tan x_{i}^{*}}=n r^{2} \cdot \tan \frac{\pi}{n}
$$


de donde:

$$
\alpha\left(S\left(P_{6}^{*}\right)\right)=2 r^{2} \sqrt{3}<4 r^{2}=\alpha\left(P_{4}^{*}\right)<3 r^{2} \sqrt{3}=\alpha\left(S\left(P_{3}^{*}\right)\right)
$$

b) Las células de los panales son construidas en secciones así como se ve en la Figura 1. Podrían existir otras configuraciones, por ejemplo la de la Figura 4. Las abejas prefieren la primera configuración, se ve que el módulo de resistencia de una célula de la primera es algo más grande que el módulo de resistencia de una célula de la segunda configuración:

$$
W^{\prime}=\frac{5}{8} \cdot r^{3}=0.625 \cdot r^{3} m^{3}
$$

es algo más grande que el módulo de resistencia de una célula de la segunda configuración:

$$
W^{\prime \prime}=\frac{5 \sqrt{3}}{16} \cdot r^{3}=0.540 \cdot r^{3} m m^{3}
$$

(ver [10], pág. 946-948).

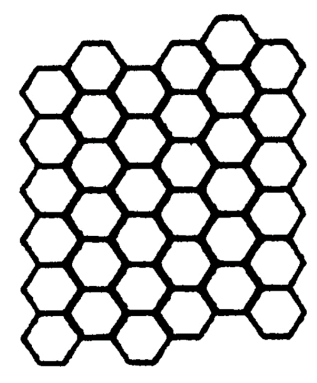

Figura 4: Otras configuraciones de panales.

Las configuraciones de las secciones precedentes ahora dan una solución al problema A de la introducción: mejorando siempre el proyecto inicial de la construcción de los panales, en decenas miles de años de búsqueda del óptimo, las abejas renunciaron al contorno de las células para eliminar los huecos, adoptaron el contorno poligonal circunscrito al contorno circular del cuerpo de la abeja, luego pasaron al contorno poligonal regular con un mismo número de lados para minimizar el consumo de cera en el proceso de construcción de las células (teorema 4.3); a continuación para realizar una construcción perfecta de todo el panal, las abejas se vieron en la necesidad de admitir que los polígonos podían ser solamente triángulos equiláteros, cuadrados o hexágonos regulares (teorema 5.1) pero, como entre las áreas de las superficies limita.das de las últimas tres figuras, y entre las áreas laterales de las células prismáticas correspondientes, la menor es el área de la superficie limitada por el hexágono (teorema 5.2), las abejas adoptaron, finalmente por el contorno hexagonal regular. 
I. MUNTEAN

\section{Referencias}

[1] Carruccio, E. (1936) "Notizie storiche sulla geometría delle api", Periódico di Matematiche 16(4): 35-54.

[2] lonescu, I. (1955) Maxime si Minime Geometrice, 2a Ed. Editura Tehnica, Bucuresti.

[3] Kepler, J. (1611) Sirena seu de Nive sexángula (En latín). Godefrindum Tambach. Erancofurti ad Moenum. (Ver: Kepler: Opera Ominia, Vol. VII, pag. 715-730. Ed. Ch. Frisen, Heyder fe Zimmer, Frankfurt a. M. fe Eriangen, 1868).

[4] Máraldi, G.F. (1712) "Observations sur les abeilles", Mémoires de l'Académie Royale des Sciences, París: 299-335.

[5] Muntean, I. "Problema lui Réaumur privind constructia optimalá a fagurilor de albina" preprint.

[6] Nemytskii, V.V. et alii. (1957) Lecţii de Analiza Matematică, Vol. II, 2a Ed. (1a rusa). Gos. Izdat. Tekhniko-Teoret. Lit., Moscú.

[7] Nicolescu, M. et alii. (1980) Analiza Matematică. Vol. I, 5a Ed. Ed. Didáctica si Pedagógica, Bucuresti.

[8] Pappus de Alejandría (1933) La Collection Mathématique. Éd. Desdée de Brouwer et Cie., Paris et Bruges.

[9] Pinch, E.R. (1993) Optimal Control and Calculus of Variations. Oxford University Press, Oxford.

[10] Posea, N.; Anghel, Al.; Manea, C.; Hotea, Gh. (1986) Rezistenta Materialelor. Probleme. Ed. Ştiinţific şi Enciclopedic, Bucuresti.

[11] Steinmetz, H. (1930) "Bemerkungen zu: Johannes Kepler, Strena seu de nive sexangula", in: K. Stöckl (Ed.) Kepler-Festschrift, Johannes Kepler, der kaiserliche Mathematiker, Vol. 1 Bericht des Naturwissenschaftlicher Verein, Regensburg: 257-263.

[12] Togliatti, E.G. (1938) "Massimi e minimi", in: Enciclopedia delle matematiche elementari, Vol. II, Hoepli, Milano: 1-71.

[13] Vogt. H. (1911) Geometrie und Ökonomie der Bienenzelle. Trewendt Granier, Bres.

[14] Volcinschi, T. (1988) Ceara. Redactia de Propaganda Tehnica Agricola, Bucuresti.

[15] Willem, V. (1928, 1930) "L'architecture des abeilles", Académie Royale de Belgique, Bulletin de la Classe des Sciences 14(5) (1928), 672-705; 16 (1930): 893-906.

[16] Periódico Adevárui de Bucuresti, 20 mai 1995. 


\section{Obituario}

El profesor Ioan Muntean murió el 16 de agosto de 1996. Durante los años 1983- 1996 fue colaborador nuestro y publicó varios trabajos en Costa, Rica. Sus campos de interés fueron: análisis funcional, control óptimo y optimización. Autor de más de 120 trabajos en estos campos, incluyendo varios libros, el profesor Muntean deja abiertas interesantes líneas de investigación:

- Lemaire, B.; Muntean, I. (1961) "Évaluation des cycles limites de certains systémes différentiels", Revue Française de Traitement de l'Information 6: 255-274.

- Muntean, I. (1968) "Harmonic oscillations for some systems of two differential equations", J. Math. Anal. Appl. 24: 474-485.

- Cobzaş, Ş.; Muntean, I. (1981) "Condensation of singularities and divergence results in approximation theory", J. Approx. Theory., 31(2): 138-153 (con ).

- Muntean, I. (1983) "Simultaneous optimization of maintenance and replacement policy for machines", Problems of Control and Information Theory 12(4): 279-293.

- Muntean, I. (1987) "Some transcendent elementary functions", Matematikai Lapok. 92: $419-425$.

- Muntean, I.; Vornicescu, N. (1994) "Estrategias de aprendizaje en tiempo mínimo", Métodos Matemáticos Aplicados a las Ciencias, VII y VIII Simposio, Editorial de la U.C.R.: 182-201. 